\title{
Burden of migraine related to menses: results from the AMPP study
}

Jelena M Pavlović ${ }^{1,2^{*}}$, Walter F Stewart ${ }^{3}$, Christa A Bruce ${ }^{3}$, Jennifer A Gorman ${ }^{4}$, Haiyan Sun ${ }^{4}$, Dawn C Buse ${ }^{1,2}$ and Richard B Lipton ${ }^{1,2}$

\begin{abstract}
Background: Studies of the difference between menstrually associated and non-menstrually associated migraine are somewhat controversial. The majority of studies have focused on comparing menstrual to non-menstrual attacks rather than comparing study groups with different migraine diagnoses with respect to menstruation. As there is limited knowledge available on the overall impact and burden of migraine among groups of women with and without menstrually associated migraine our goal was to examine differences between these groups. We hypothesized that there would be greater burden of migraine related to menstruation and headache frequency in a population study across groups of women.
\end{abstract}

Methods: We analyzed data from the American Migraine Prevalence and Prevention (AMPP) Study, a longitudinal, US, population-based study. We included female respondents to the 2009 survey, aged 18 to 60 , who met modified ICHD-2 criteria for migraine, were actively menstruating and fit one of three definitions based on the self-reported association of menses and migraine attacks: self-reported predominantly menstrual migraine (MM, attacks that only or predominantly occur at the time of menses), self-reported menstrually-associated migraine (MAM, attacks commonly associated with menses, but that also occur at other times of the month), and self-reported menstrually-unrelated migraine (MUM). These three groups were compared on characteristics and measures of headache impact and burden (Headache Impact Test- 6 item (HIT-6) and Migraine Disability Assessment Scale (MIDAS).

Results: There were 1,697 eligible subjects for this study in the following categories: MM (5.5\%), MAM (53.8\%), or MUM (40.7\%). Women with MM had an older age of migraine onset. Those with predominantly menstrually-related attacks (MM) had fewer headache-days but appeared to be more impaired by attacks. HIT-6 and MIDAS scores were significantly higher for both the MM and MAM groups compared with the MUM groups; however, effects were more robust for MM than MAM.

Conclusions: Nearly $60 \%$ of women with migraine reported an association between migraine and menses. These women reported greater headache impact and migraine-related burden on functioning than those in whom migraines were not related to menstruation. Women with MM were more impaired by attacks while women with MAM had overall highest burden, likely due to experiencing migraines on additional days.

Keywords: Migraine; Disability; Burden; Prevalence; Menstrual cycle; Menstrual migraine

\footnotetext{
* Correspondence: jpavlovi@montefiore.org

'Department of Neurology, Albert Einstein College of Medicine, Bronx, NY, USA

${ }^{2}$ Montefiore Headache Center, Bronx, NY, USA

Full list of author information is available at the end of the article
}

\section{国 Springer}

(c) 2015 Pavlovic et al.; licensee BioMed Central. This is an Open Access article distributed under the terms of the Creative Commons Attribution License (http://creativecommons.org/licenses/by/4.0), which permits unrestricted use, distribution, and reproduction in any medium, provided the original work is properly credited. 


\section{Background}

Many women with migraine report an association between migraine attacks and menses. These attacks have been referred to as "menstrual" migraine by both patients and clinicians and have been the focus of an evolving definition of Menstrual Migraine by the International Classification of Headache Disorders (ICHD-2) $[1,2]$. The proportion of women with menstrually-related migraine varies among studies from 3\% [3] to 76\% [4], depending upon study populations and diagnostic criteria used. The use of broad encompassing case criteria may mask a severity effect, especially if the effect is confined to a more narrowly defined menstrual migraine subgroup. Clinical experience and results from clinic based studies support that peri-menstrual migraine attacks are more severe [3,5-7], longer in duration [3,8-10], have greater associated impairment $[9,10]$ and are more difficult to treat $[3,8,10]$ than migraine attacks at other times of the month.

There has been a long-standing preconception that this severity of menstrually associated migraines shown in clinical studies has not been seen in population-based studies [11-13]. The common explanations for this have been the selection bias in specialty clinics and the outcomes that were measured to represent dimensions of severity (e.g., pain intensity, pain duration, symptoms, disability, etc). Lack of precise menstrual migraine diagnoses and frequent variability in interpretation of the diagnoses among researchers has added to the confusion. Furthermore, the majority of prior population and clinic-based studies comparing menstrual to nonmenstrual migraine have been based on prospective diaries and have focused on comparing menstrual attacks to non-menstrual attacks $[6,10,11,14,15]$, rather than comparing different groups of migraineurs [3]. Therefore, it was the goal of this study to examine the severity and associated burden/impact of migraine across groups that are variously affected by menstruation, rather than only across attacks. The diagnostic criteria for menstrual migraine are still evolving and remain in the appendix of the ICHD-3beta [2] with the requirement for prospective documentation of the association of migraine attack and menses with three months of diary data. Since no population study has obtained three month menstruation related diary data on more than a hundred women, there is value in examining subgroups of women based on their self-reported relationship of migraine to menstruation as commonly occurs in clinical settings.

In the present study, we used self-reported data from the 2009 American Migraine Prevalence and Prevention (AMPP) Study [16] to define three groups of women with migraine using criteria and definitions that are different, but in spirit consistent with the ICHD criteria [2] (Table 1): self-reported predominantly menstrual
Table 1 Headache and menstrual cycle definitions

American Migraine Prevalence and Prevention Study, 2009

Self-reported definitions used in this paper

Self-reported predominantly

Menstrual migraine (MM)

Headaches in relation to your period: "all of my headaches are related to my menstrual cycle"(my headaches ONLY happen around the time of my period (from two days before to three days after the start of menstruation)

Self-reported Menstruallyassociated migraine (MAM)

Headaches in relation to your period: "I am more likely to get headaches with my period but they also occur at other times of the month"

Self-reported Menstruallyunrelated migraine (MUM)

Headaches in relation to your period: "my headaches are not related to my menstrual cycle"

International Headache Society (ICHD-3beta) [2]

\section{Diagnostic Criteria}

Pure menstrual migraine

Documented and prospectively collected evidence that attacks occur exclusively on day $1 \pm 2$ (i.e., days -2

to +3$)^{1}$ of menstruation in at least two out of three menstrual cycles and at no other times of the cycle

Menstrually-related migraine

Documented and prospectively collected evidence that attacks occur on day $1 \pm 2$ (i.e., days -2 to +3$)^{1}$ of menstruation in at least two out of three menstrual cycles and additionally at other times of the cycle

Non-menstrual migraine

Attacks have no menstrual relationship

1 - The first day of menstruation is day 1 and the preceding day is day -1 ; there is no day 0.

migraine (MM) for headaches that occur with menses (two days before to three days after the start of menstruation), self-reported menstrually-associated migraine (MAM) for headaches associated with menses, but that also occur at other times of the month, and self-reported menstrually-unrelated migraine (MUM). We then compared these three groups to determine if there was variation in the impact (Headache Impact Test- 6 item [HIT-6]) and migraine-related disability (Migraine Disability Assessment Scale [MIDAS]). We hypothesized that the association with menses will contribute to significant burden of migraine and that women with MAM will experience the most significant burden because they have menstrually-related attacks as well as attacks outside of the menstrual window.

\section{Methods}

The AMPP Study was approved by the Albert Einstein College of Medicine Institutional Review Board. The AMPP Study was modeled on the methods of the American Migraine Studies I [17] and II [18], described in detail elsewhere [16] and briefly summarized herein. In 2004, a screening survey was mailed to a stratified random sample of 120,000 U.S. households encompassing 
257,339 household members [16]. The questionnaire was completed by the head of the household, who reported the total number of household members and the number of household members experiencing at least occasional self-defined "severe headache". Each household member with severe headaches was asked to complete a questionnaire that included questions the AMS/AMPP diagnostic module which allows for assignment of migraine based upon on modified ICHD-2 criteria. This module has been demonstrated to have a sensitivity of $100 \%$ and specificity of $82 \%$ for the diagnosis of migraine [19]. No significant changes occurred between ICHD- 2 and ICHD-3beta that are related to the criteria used in this study.

A random sample of 24,000 of the adult (i.e., $\geq 18$ years of age) respondents to the 2004 survey who self-reported active (i.e., past 12 months) "severe headache" were asked to participate in an annual longitudinal follow up study. They completed questionnaires annually from 2005-2009. We used the 2009 AMPP Study survey because complete data were available to describe subtypes of menstrual migraine and it included the HIT-6 (with paid permission for use) as well as the MIDAS.

In the 2009 survey, respondents answered questions about headache symptoms, frequency, severity, menstrual status and whether they had headache/migraine associated with menstruation, employment status (i.e., working for pay full- or part-time, unemployed, retired, a student, a homemaker, disabled, a volunteer, on medical or maternity leave, or "other") and completed the MIDAS [20] and HIT-6 questionnaire [21]. 17,052 females were mailed the 2009 AMPP Study questionnaire and $71.4 \%(\mathrm{n}=12,180)$ were completed and returned (Figure 1).

Respondents were asked to indicate the number of days they had headache in the preceding three months and the number of days with their "most severe type of headache" over the preceding year. A total of 5,134 female respondents of the 2009 AMPP Study survey met criteria for episodic migraine and were considered active cases (i.e., defined as having at least one migraine in the previous 12 months and an average of $<15$ headache days per month over the preceding 3 months). Of these, 1,697 were eligible for this analysis which included the following: aged 18 to 60 years, had at least one menstrual cycle in the preceding two months, and responded to the questions used to determine menstrual migraine status.

\section{Menstrual migraine subgroups}

The diagnostic criteria for migraine in association with menses have not been firmly established; as a consequence in ICHD-3beta [2] (Table 1) the criteria remain in the Appendix, indicating that they are not yet fully accepted, but intended for further study. We could not apply ICHD-3beta criteria as written because we relied on self-reported recall of headaches associated with menses, a common approach both in large scale epidemiological studies and in clinical care. Our large scale study does not include daily diaries as recommended by ICHD-3beta criteria in order to establish a diagnosis.

The AMPP Study questionnaire asked, "Which statements best describe your headaches in relation to your period?" Respondents were classified into three mutually exclusive groups based on their responses. Women who selected "all of my headaches are related to my menstrual cycle (my headaches ONLY happen around the time of my period from two days before to three days after the start of menstruation)" were assigned to the self-reported predominantly menstrual migraine (MM) group. Women who selected "I am MORE LIKELY to get headaches with my period but they also occur at other times of the month" were assigned to the self-reported menstrually-associated migraine (MAM) group unless they also endorsed the MM statement. Finally, women who selected " $m y$ headaches are NOT related to my menstrual cycle" were assigned to the self-reported menstrually-unrelated migraine (MUM) group if they did not also endorse the MM or MAM statement. Another difference between how we defined our subgroups and ICHD-3beta criteria is that we did not require women to endorse "I get headaches with at least two of every three periods" as these data were not collected.

We did not exclude women who endorsed more than one response option. Of respondents who endorsed the response "My headaches ONLY happen around the time of my period [from two days before to three days after the start of menstruation]" about one third also endorsed "I am MORE LIKELY to get headaches with my period but they also occur at other times of the month". Simultaneous endorsement of both statements most likely occurs in women who have predominantly MM with occasional migraine and/or headaches at other times. Accordingly, we assigned these women to the self-reported predominantly MM group (MM). This decision and its potential consequences are considered in the discussion. "I am MORE LIKELY to get headaches with my period but they also occur at other times of the month" and "my headaches are NOT related to my menstrual cycle" were both endorsed by $4.1 \%$ of the cases and these women were assigned into the MAM group. This is likely conservative since these cases likely decreased the strength of observed associations.

\section{Number of migraine attacks and days with migraine/ headache}

Headache attack frequency was based on a response to a question about the number of days with headache (for most severe type of headache) in the past month, three 


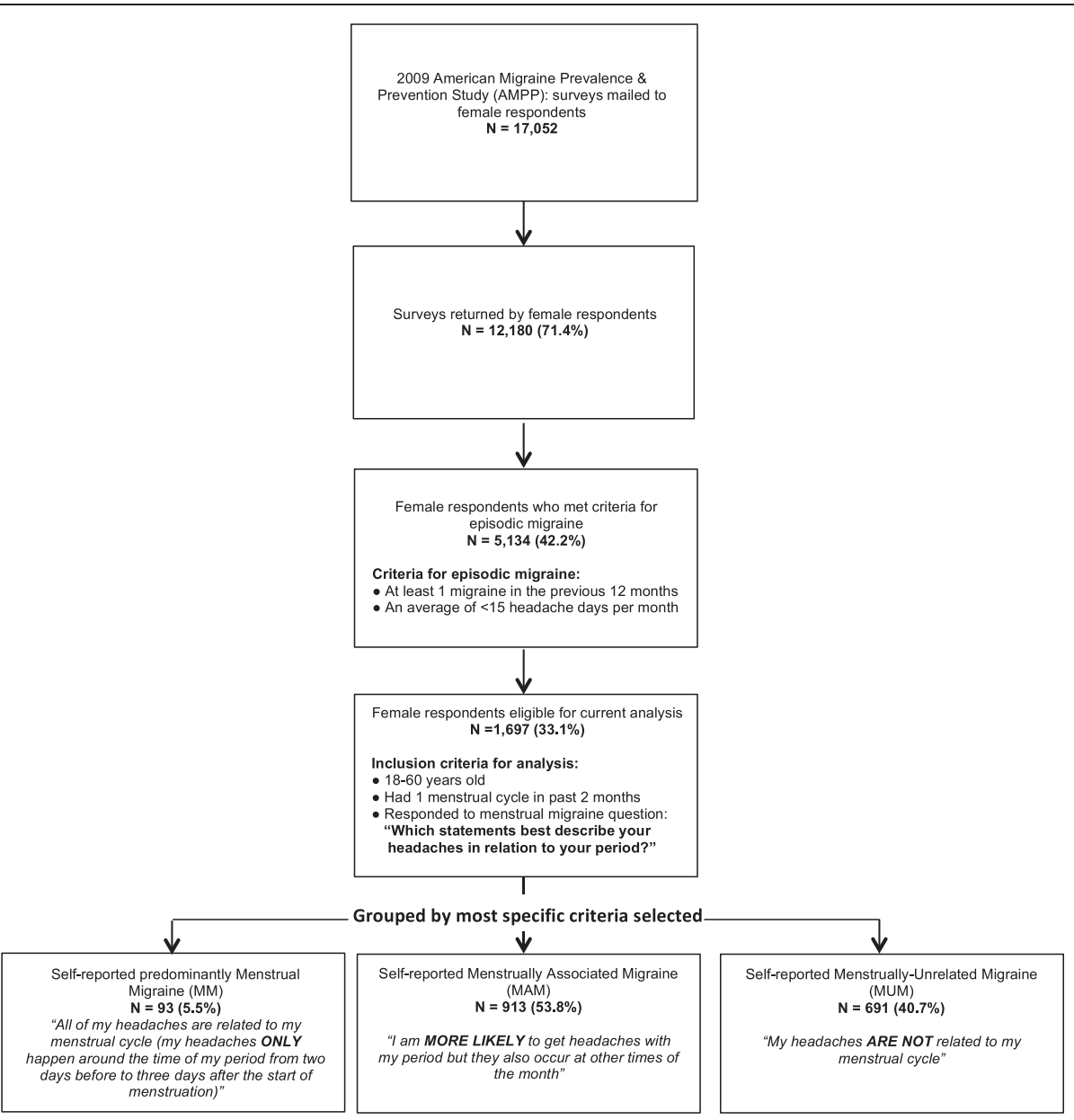

Figure 12009 AMPP female respondents and group assignment.

months, and 12 months and converted to a measure of headaches per month. Headache-days in the past three months was taken from the response to a MIDAS question on the number of days with headache in the past three months (one migraine attack could last for multiple days).

\section{Headache impact on functioning}

We used the following migraine/headache impact measures: MIDAS (scored by grade), a MIDAS measure of lost productive time (LPT) [22,23] and the HIT-6 [21].

\section{Migraine-related disability}

The MIDAS is comprised of five questions related to impact of migraine/headache on functioning in defined roles (i.e., housework, work for pay, leisure time) [20] that are used to categorize severity into four grades based on total days of impaired functioning (i.e., Grade I or little or no disability, 0 to 5; Grade II or mild disability, 6 to 10; Grade III or moderate disability, 11 to 20;
Grade IV or severe disability, $\geq 21$, with an option of dividing Grade IV into IVA and IVB). The recall period for the MIDAS is three months, effectively covering three menstrual cycles.

\section{Lost productive time}

We used the two MIDAS work-for-pay questions to estimate lost productive time (LPT). These two questions ask about number of days of missed work in the past three months due to headache (absenteeism) and number of days while at work where productivity was reduced by half (presentism denoted as Half Days) or more because of headache.

\section{Employment}

Employment status was documented [22] for each respondent and defined as actively employed for pay (i.e., excludes those on short- or long-term medical leave) if they reported actively working enough hours to qualify for full- ( $\geq 35$ hours) or part-time (20 to 34 hours) status 
in the previous two weeks. Individuals were defined as eligible for employment but "unemployed" if they reported they were either unemployed or on medical disability. For analysis, employment was defined as a binary variable (i.e., employed versus unemployed).

We also used the HIT-6, (with a licensing fee) which is comprised of six questions, to assess the impact of the most severe headache type on ability to function. Three questions are independent of headache frequency and three questions are related to frequency of headache attacks. It has a recall period of four weeks [21]. It should be noted that both MIDAS and HIT-6 impact measures do not specifically assess headache or migraine days related to menstruation, but all headache-days over an interval of 3 months and 1 month, respectively.

\section{Analyses}

The analysis was confined to women who both met criteria for migraine and had menstrual cycles in the twomonth period before completing the 2009 AMPP Study survey. We compared the three subgroups defined by self-reported menstrual headache status (i.e., MM, MAM, MUM) on characteristics and measures of headache impact.

LPT was defined as: Missed Work Days $+0.5^{*}$ (Workdays Where Productivity was Reduced by Half)

The LPT formula is for the number of missed workday equivalents in the three months before the survey. We assumed that a workday reduced by half or more is equivalent to working for half of a usual workday, an estimate that was validated in a previous diary study [20]. Based on previous experience $[22,23]$ and given the nonnormal distribution properties of work loss (i.e., a substantial proportion with no work loss), LPT was defined as two binary variables (i.e., $<0.5$ LPT Days versus $0.5+$ LPT days and $<2$ LPT Days versus 2+ LPT days). For analysis, employment was defined as a binary variable (i.e., employed versus unemployed). We applied the standard scoring algorithm for HIT-6 items where responses are assigned values (i.e., Never $=6$, Rarely $=8$, Sometimes $=10$, Very Often $=11$, Always $=13$ ) and summed for a total score. We stratified respondents using the standard cut-points. (i.e., $<50=$ little or no impact, and 50 to 55 for some impact, 56 to 59 for substantial impact, $\geq 60$ for very severe impact). We completed regression analysis using cut-points above and below 56 (i.e., $<56, \geq 56$ ) and 60 (i.e., $<60, \geq 60$ ).

We used chi-square to compare the sociodemographic and headache characteristics of the three subgroups, and we used logistic regression (i.e., LPT, HIT-6 item variables) to analyze the relationship between menstrual migraine status and measures of headache impact, as previously defined. We completed separate logistic regression models for women who reported six or fewer headache-days in the past three months (i.e., an average of two headache-days per month) and for all women with EM (i.e., $\leq 45$ headache-days in three months). Covariates were added in two sequential models that included: 1 ) age (18 to 29,30 to 39,40 to 49,50 to 60 ); race (White, Black, Other); education (no high school diploma, high school diploma, some college or associates degree, bachelors degree, graduate degree); annual household income $(<\$ 22,500, \$ 22,500$ to $\$ 39,999, \$ 40,000$ to $\$ 59,999, \$ 60,000$ to $\$ 89,999, \geq \$ 90,000$ ), and age at migraine onset; and 2) number of attacks of the most severe type of headache in the past month. SAS version 9.2 was used for all analyses (SAS Institute Inc., Cary, NC).

\section{Results}

Among the 1,697 respondents who met inclusion criteria (Table 2), 5.5\% ( $\mathrm{n}=93)$ were categorized as MM, 53.8\% $(\mathrm{n}=913)$ as MAM, and $40.7 \%(\mathrm{n}=691)$ as MUM using the criteria presented in Table 1. Overall, the three groups significantly differed by education, annual household income, number of headache types, and age of onset of migraine. Those in the MAM group tended to have a higher education level, whereas those in the MM group tended to have a higher income. Women with MM had fewer headache types and a later age of migraine onset (i.e., $34.4 \%$ on or after age 30 versus $<24.0 \%$ for the other two groups). Menstrual migraine association status was not associated with other covariates. The number of headache-days in the past three months differed significantly among the three groups (Table 3 ). In the MM group, $68.8 \%$ of cases had six or fewer headache-days in the past three months compared to 53.3\% for MAM cases and $61.7 \%$ for MUM cases ( $<20.001)$. In the MAM group, persons with $\geq 15$ headache-days in the past three months were more common $(p<0.001)$. The distribution by number of headache attacks is difficult to compare by group because of the variation in percent with unknown values. In particular, $17.2 \%$ of MM cases had an unknown value. Furthermore, the difference in distribution by number of headache-days is an artifact, in part, of the case definition for MM (i.e., "my headaches ONLY happen around the time of my period [from two days before to three days after the start of menstruation]").

\section{Headache impact}

Impact measures, which were dependent on headachedays, also differed by menstrual migraine status. Specifically, the distribution by the four MIDAS Grades varied significantly by menstrual migraine status (chi-square $\mathrm{p}<0.001)$. Proportionately more MAM $(23.8 \%)$ and MM (16.1\%) cases had MIDAS scores which classified them as Grade III or IV (moderate or severe) compared to MUM (15.4) cases. The unemployment rate (i.e., looking for work but not employed/[looking for work + employed]) 
Table 2 Sociodemographic and headache characteristics by menstrual group in women with episodic migraine who are actively menstruating: results from the AMPP 2009 Survey

All $(\mathrm{N}=1,697)$

\begin{tabular}{ccc} 
& Stratified by Menstrual Group \\
\hline Self-reported & Self-reported & Self-reported \\
predominantly & Menstrually & Menstrually \\
Menstrual Migraine & associated migraine & unrelated migraine \\
$(\mathrm{MM})(\mathrm{N}=93)$ & (MAM) $(\mathrm{N}=913)$ & (MUM) $(\mathrm{N}=691)$
\end{tabular}

Age, years

$<30$

30-39

40-49

50-60

Race

White

Black

Asian, Pacific Islander

Other

Unknown

Education ${ }^{1}$

High school grad or less

Some college

Bachelor degree

Graduate degree

Unknown

Current Employment Status ${ }^{2}$

Full-time

Part-time

Unemployed

Other

Annual Household Income ${ }^{3}$

$<\$ 22,500$
$\$ 22,500-\$ 39,999$
$\$ 40,000-\$ 59,999$
$\$ 60,000-\$ 89,999$
$\geq \$ 90,000$

Body Mass Index

Underweight

Normal

Overweight

Obese

Headache Types ${ }^{4}$

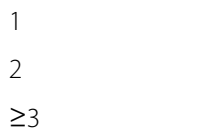

Unknown

$\begin{array}{ll}196 & 11.6 \% \\ 554 & 32.6 \% \\ 748 & 44.1 \% \\ 199 & 11.7 \%\end{array}$

118

$88.1 \%$

$7.0 \%$

$1.6 \%$

$27 \quad 1.6 \%$

29

$1.7 \%$

$375 \quad 22.1 \%$

$412 \quad 24.3 \%$

$639 \quad 37.6 \%$

$240 \quad 14.1 \%$

$31 \quad 1.8 \%$

958

$56.2 \%$

$16.1 \%$

248

218

$14.3 \%$

$13.2 \%$

248

321

403

$14.6 \%$

$18.9 \%$

$23.7 \%$

$19.5 \%$

$23.2 \%$

$81 \quad 4.8 \%$

$571 \quad 33.6 \%$

$431 \quad 25.4 \%$

$614 \quad 36.2 \%$

$455 \quad 26.8 \%$

$772 \quad 45.5 \%$

$413 \quad 24.3 \%$

$57 \quad 3.4 \%$

$10.7 \%$
$23.7 \%$
$47.3 \%$
$18.3 \%$

103

289

410

111

$82.8 \%$

$11.8 \%$

$2.2 \%$

$1.1 \%$

$2.2 \%$

$26.9 \%$

$23.7 \%$

$31.2 \%$

$15.1 \%$

$3.2 \%$

$50.5 \%$

$23.7 \%$

$14.0 \%$

$11.8 \%$

$17.2 \%$

$17.2 \%$

$19.4 \%$

$17.2 \%$

$29.0 \%$

$1.1 \%$

$29.0 \%$

$30.1 \%$

$39.8 \%$

$45.2 \%$

$34.4 \%$

$12.9 \%$

$7.5 \%$
(MAM) $(\mathrm{N}=913)$ (MUM) $(\mathrm{N}=691$ ) 
Table 2 Sociodemographic and headache characteristics by menstrual group in women with episodic migraine who are actively menstruating: results from the AMPP 2009 Survey (Continued)

\begin{tabular}{|c|c|c|c|c|c|c|c|c|}
\hline \multicolumn{9}{|c|}{ Age of Onset - most severe headache ${ }^{5}$} \\
\hline$<15$ & 351 & $20.7 \%$ & 13 & $14.0 \%$ & 218 & $23.9 \%$ & 120 & $17.4 \%$ \\
\hline $15-19$ & 316 & $18.6 \%$ & 15 & $16.1 \%$ & 182 & $19.9 \%$ & 119 & $17.2 \%$ \\
\hline $20-24$ & 272 & $16.0 \%$ & 5 & $5.4 \%$ & 143 & $15.7 \%$ & 124 & $17.9 \%$ \\
\hline $25-29$ & 190 & $11.2 \%$ & 11 & $11.8 \%$ & 97 & $10.6 \%$ & 82 & $11.9 \%$ \\
\hline$\geq 30$ & 384 & $22.6 \%$ & 32 & $34.4 \%$ & 192 & $21.0 \%$ & 160 & $23.2 \%$ \\
\hline Unknown & 184 & $10.8 \%$ & 17 & $18.3 \%$ & 81 & $8.9 \%$ & 86 & $12.5 \%$ \\
\hline
\end{tabular}

1 - Chi -square $p=0.035$ for education level, when include unknowns $p=0.064$.

2 - Chi-square $\mathrm{p}=0.019$ for employment status; "Unemployed" encompasses reported unemployed and those on medical leave. "Other" includes homemaker,

retired, student, volunteer and other. Self-employed was included in the full time employed group.

3 - Chi-square $p=0.028$ for household income.

4 - Chi-square $p<0.001$ for headache type (including/excluding unknowns).

5 - Chi-square $p=0.001$ for age of onset of most severe headache type (including/excluding unknowns).

differed ( $\mathrm{p}=0.019)$, with the highest rate occurring among MM cases (17.7\%) compared to the other two groups (12.4\% to $14.0 \%)$. In univariate analysis, only distribution by the MIDAS LPT measure differed among the three migraine groups $(\mathrm{p}<0.001)$ (Table 3$)$.

The three migraine subgroups differed by age, headache-days, and other factors that could confound the comparison on measure of functional impact. We completed logistic regression models (i.e., comparing MM and MAM cases to MUM cases) using measures of headache impact as the outcome for respondents with six or fewer headache-days in the past three months (58.5\% of all cases and $68.8 \%$ of MM cases) and then for all EM cases (headache-days frequency per three months <45) (Table 4). The odds ratios did not differ for those with six or fewer headache-days/three months compared to all EM cases. We also adjusted for all covariates except for headache attack frequency (Model I) and then again by including headache attack frequency (Model II). The odds ratios were similar for models without (Model I) and with (Model II) adjustment for headache attacks in the previous month. Among those with six or fewer headache-days/three months (model 1 ), statistically significant elevated odds ratios were observed for MIDAS LPT (OR = 2.4, 95\% CI: 1.1-5.1) and for a HIT-6 score of $56+(\mathrm{OR}=2.5$; 95\% CI: 1.3-4.7) among MM cases $(\mathrm{p}<0.01)$. Odds ratios for MAM cases were consistently lower than that of MM cases and were less often statistically significant.

\section{Discussion}

To assess burden of migraine in the population, we compared three groups of women with migraine based on the self-reported association of their migraine attacks with menses using data from the AMPP Study. The majority of women reported some association of migraine with menses, though in most, migraine also occurred at other times of the month. Those with predominantly menstrually-related attacks (MM) had fewer headache-days but appeared to be more impaired by attacks as measured by impairment in ability to work and elevated HIT-6 scores. Women with MAM had overall highest burden, due to experiencing not only peri-menstrual migraines but additional migraines outside of the peri-menstrual window.

Women with MM had fewer headache-days per three months, as was expected given the case definition for MM. In contrast, the MAM group had the highest number of headache-days per three months. This pattern may be important when interpreting findings for the MIDAS LPT headache-days adjusted measure and the HIT-6 measure (Table 4). These measures reflect, in part, the frequency of headaches, as well as, the impact that headaches have on functioning. The adjusted odds ratios for MIDAS LPT were statistically significant and indicate that women with MM experience headaches that have a substantial impact on the work role that is more disabling than that of the other two subgroups. Moreover, as the threshold for work impact was increased (i.e., missed days of work or absenteeism), the difference between MM cases and other cases increased, a finding that is consistent with specialty clinic $[9,10]$ and population studies $[3,24]$. The HIT-6 measures the global impact of headaches on the social role and other domains of functioning and includes a measure of pain. The elevated odds ratios for a "severe impact" HIT-6 score $(\geq 56)$ is consistent with the above findings for MIDAS and suggests a more general impact on functioning from MM than the work role.

Previous diary studies compared all peri-menstrual headaches to headaches at other times in the cycle. The prior study from our group based on prospectively filled diaries from the general population [11] and one clinic study suggested that attacks of menstrual and nonmenstrual migraine have similar disability [25]. However, other studies primarily from specialty clinics, suggest 
Table 3 Headache experience and impact by menstrual group: results from the AMPP 2009 survey

\begin{tabular}{|c|c|c|c|c|c|c|c|c|}
\hline \multirow[t]{2}{*}{ Measure } & \multirow{2}{*}{\multicolumn{2}{|c|}{ All $(\mathrm{N}=1,697)$}} & \multicolumn{6}{|c|}{ Stratified by menstrual group } \\
\hline & & & \multicolumn{2}{|c|}{$\begin{array}{l}\text { Self-reported } \\
\text { predominantly } \\
\text { Menstrual Migraine } \\
(\mathrm{MM})(\mathrm{N}=93)\end{array}$} & \multicolumn{2}{|c|}{$\begin{array}{c}\text { Self-reported } \\
\text { Menstrually } \\
\text { associated migraine } \\
\text { (MAM) }(\mathrm{N}=913)\end{array}$} & \multicolumn{2}{|c|}{$\begin{array}{c}\text { Self-reported } \\
\text { Menstrually } \\
\text { unrelated migraine } \\
(\mathrm{MUM})(\mathrm{N}=691)\end{array}$} \\
\hline \multicolumn{9}{|c|}{ Headache-Days in the Past 3 months (MIDAS item) ${ }^{1}$} \\
\hline $0-3$ & 605 & $35.6 \%$ & 37 & $39.8 \%$ & 271 & $29.7 \%$ & 297 & $43.0 \%$ \\
\hline $4-6$ & 371 & $21.9 \%$ & 27 & $29.0 \%$ & 215 & $23.6 \%$ & 129 & $18.7 \%$ \\
\hline $7-10$ & 299 & $17.6 \%$ & 12 & $12.9 \%$ & 180 & $19.7 \%$ & 107 & $15.5 \%$ \\
\hline $11-14$ & 88 & $5.2 \%$ & 4 & $4.3 \%$ & 57 & $6.2 \%$ & 27 & $3.9 \%$ \\
\hline$\geq 15$ & 306 & $18.0 \%$ & 10 & $10.7 \%$ & 179 & $19.6 \%$ & 117 & $16.9 \%$ \\
\hline Unknown & 28 & $1.6 \%$ & 3 & $3.2 \%$ & 11 & $1.2 \%$ & 14 & $2.0 \%$ \\
\hline \multicolumn{9}{|c|}{ Headache Attack Frequency in the Past Month ${ }^{2}$} \\
\hline $0-<1$ & 568 & $33.5 \%$ & 13 & $14.0 \%$ & 279 & $30.6 \%$ & 276 & $39.9 \%$ \\
\hline $1-<2$ & 563 & $33.2 \%$ & 44 & $47.3 \%$ & 324 & $35.5 \%$ & 195 & $28.2 \%$ \\
\hline $2-<3$ & 214 & $12.6 \%$ & 10 & $10.7 \%$ & 130 & $14.2 \%$ & 74 & $10.7 \%$ \\
\hline$\geq 3$ & 260 & $15.3 \%$ & 10 & $10.7 \%$ & 149 & $16.3 \%$ & 101 & $14.6 \%$ \\
\hline Unknown & 92 & $5.4 \%$ & 16 & $17.2 \%$ & 31 & $3.4 \%$ & 45 & $6.5 \%$ \\
\hline \multicolumn{9}{|l|}{ MIDAS Grade ${ }^{3}$} \\
\hline I. Little or no disability & 1057 & $62.3 \%$ & 54 & $58.1 \%$ & 533 & $58.4 \%$ & 470 & $68.0 \%$ \\
\hline II. Mild disability & 247 & $14.6 \%$ & 16 & $17.2 \%$ & 145 & $15.9 \%$ & 86 & $12.5 \%$ \\
\hline III. Moderate disability & 184 & $10.8 \%$ & 9 & $9.7 \%$ & 122 & $13.4 \%$ & 53 & $7.7 \%$ \\
\hline IV. Severe disability & 154 & $9.1 \%$ & 6 & $6.4 \%$ & 95 & $10.4 \%$ & 53 & $7.7 \%$ \\
\hline Unknown & 55 & $3.2 \%$ & 8 & $8.6 \%$ & 18 & $2.0 \%$ & 29 & $4.2 \%$ \\
\hline \multicolumn{9}{|c|}{ Lost Productive Time (LPT) (days in past 3 months) (MIDAS item) ${ }^{4}$} \\
\hline 0 & 760 & $61.9 \%$ & 34 & $49.3 \%$ & 404 & $59.9 \%$ & 322 & $66.5 \%$ \\
\hline$\leq 1.00$ & 182 & $14.8 \%$ & 10 & $14.5 \%$ & 99 & $14.7 \%$ & 73 & $15.1 \%$ \\
\hline $1.01-4.99$ & 167 & $13.6 \%$ & 12 & $17.4 \%$ & 111 & $16.5 \%$ & 44 & $9.1 \%$ \\
\hline$\geq 5$ & 59 & $4.8 \%$ & 5 & $7.2 \%$ & 37 & $5.5 \%$ & 17 & $3.5 \%$ \\
\hline Unknown & 59 & $4.8 \%$ & 8 & $11.6 \%$ & 23 & $3.4 \%$ & 28 & $5.8 \%$ \\
\hline \multicolumn{9}{|c|}{ Sum Score for 3 HIT-6 Questions ${ }^{5}$} \\
\hline$\leq 49$ : little to no impact & 263 & $15.5 \%$ & 15 & $16.1 \%$ & 123 & $13.5 \%$ & 125 & $18.2 \%$ \\
\hline 50-55: some impact & 378 & $22.4 \%$ & 17 & $18.3 \%$ & 181 & $19.9 \%$ & 180 & $26.2 \%$ \\
\hline 56-69: substantial impact & 295 & $17.5 \%$ & 9 & $9.7 \%$ & 165 & $18.1 \%$ & 121 & $17.6 \%$ \\
\hline$\geq 64$ : very severe impact & 754 & $44.6 \%$ & 52 & $55.9 \%$ & 441 & $48.5 \%$ & 261 & $38.0 \%$ \\
\hline
\end{tabular}

1- Chi-square tests $p<0.001$ for MIDAS headache-days in past 3 months with or without unknown values.

2- Chi-square tests $p<0.001$ for HA frequency in past month with or without unknown values.

3- Chi-square $p<0.001$ for MIDAS grade with or without unknown values.

4- Chi-square $p<0.001$ for MIDAS LPT with or without unknown values; restricted to full time or part time employed group $(n=1,227)$.

5 - Chi-square tests $p<0.001$ for HIT-6 sum score.

that attacks of peri-menstrual migraine are more disabling than attacks of nonmenstrual migraine [8-10]. All these studies focused on comparisons of specific migraine attacks rather than migraine groups.

In this study women with any of the menstrually-related migraine disorders reported on all of their headaches, rather than separately reporting on the impact of menstrually-related and menstrually-unrelated migraine.
If menstrually-related migraine attacks are more disabling, then our data will underestimate impact of these headaches. The extent to which impact is underestimated will depend on the ratio of the number of menstrually-related migraine to the number of migraine occurring at other times in the cycle. This "dilution" effect is likely to be less significant for MM cases. The overall self-assessment by MM cases may largely represent experience with peri-menstrual headaches 
Table 4 Adjusted odds ratios (logistic regression) and associated confidence intervals for headache impact measures comparing the two menstrual migraine groups to the menstrually-unrelated migraine group, adjusting for potential confounders in two separate models ${ }^{1,2}$

\begin{tabular}{|c|c|c|c|c|c|}
\hline \multirow[t]{4}{*}{ Outcome } & \multirow[t]{4}{*}{ Group $^{3}$} & \multicolumn{4}{|c|}{ Headache-Days Frequency (per 3 months) } \\
\hline & & \multicolumn{2}{|c|}{$<=6$} & \multicolumn{2}{|c|}{$<=45$} \\
\hline & & \multicolumn{2}{|c|}{ MODEL } & \multicolumn{2}{|c|}{ MODEL } \\
\hline & & $I^{1}$ & $1 \mathrm{II}^{2}$ & $I^{1}$ & $\|^{2}$ \\
\hline \multirow[t]{2}{*}{ Odds Ratio for MIDAS LPT ${ }^{4}(0.5+\text { Days vs }<0.5 \text { Days })^{5}$} & MM & $2.4(1.1-5.1)^{7}$ & $2.0(0.9-4.3)$ & $2.1(1.1-3.8)^{7}$ & $1.9(1.0-3.5)^{7}$ \\
\hline & MAM & $1.3(0.9-1.9)$ & $1.2(0.8-1.9)$ & $1.5(1.2-2.0)^{6}$ & $1.5(1.1-1.9)^{6}$ \\
\hline \multirow[t]{2}{*}{ Odds Ratio for MIDAS LPT ${ }^{4}$ (2+ Days vs $<2$ Days) ${ }^{5}$} & MM & $5.4(1.8-15.7)^{6}$ & $5.1(1.7-15.3)^{6}$ & $2.6(1.2-5.5)^{7}$ & $2.7(1.2-5.7)^{7}$ \\
\hline & MAM & $1.8(0.9-3.7)$ & $1.6(0.8-3.4)$ & $1.9(1.3-2.9)^{6}$ & $1.8(1.2-2.6)^{6}$ \\
\hline \multirow[t]{2}{*}{ Odds ratio for HIT-6 sum score of $56+$ vs $<56^{8}$} & MM & $2.5(1.3-4.7)^{7}$ & $1.7(0.9-3.3)$ & $2.0(1.1-3.3)^{7}$ & $1.9(1.1-3.3)^{7}$ \\
\hline & MAM & $1.4(1.1-1.9)^{6}$ & $1.3(0.9-1.8)$ & $1.5(1.2-1.9)^{6}$ & $1.4(1.1-1.8)^{6}$ \\
\hline \multirow[t]{2}{*}{ Odds ratio for HIT- 6 sum score of $60+$ vs $<60^{9}$} & MM & $4.0(2.1-7.3)^{6}$ & $2.9(1.5-5.5)^{6}$ & $3.0(1.8-4.9)^{6}$ & $2.9(1.7-5.1)^{6}$ \\
\hline & MAM & $1.4(1.0-1.9)^{7}$ & $1.4(0.9-1.9)$ & $1.6(1.3-2.0)^{6}$ & $1.5(1.2-2.0)^{6}$ \\
\hline
\end{tabular}

1-Includes age, race, education level, household income and age of migraine onset as covariates.

2 - Includes the covariates from footnote " 1 " and headache attack frequency per month.

3- $\mathrm{MM}=$ self-reported predominanty menstrual migraine, $M A M=$ self-reported menstrually-associated migraine, MUM = self-reported menstrually- unrelated migraine.

4-Lost Productive Time.

5-Restricted modeling: removed 'other' employed and MIDAS LPT models only includes those full time or part time employed.

6- Indicates migraine group significance using Type III test at P-value $<0.01$.

7-Indicates migraine group significance using Type III test at P-value $<0.05$.

8 - This cut-score divides individuals with some impact or less from those with substantial impact or more.

9 - This cut-score divides individuals with substantial impact or less from those with very severe impact or more.

In bold, values of statistical significance.

in contrast to the MAM and MUM groups who are largely reporting on non-peri-menstrual headaches. It is of interest that one third of our MM cases also endorsed that they have headaches outside of their menstruation $(-2$ to +3 days from start on menstruation window), further diluting the MM subgroup and the likely burden of migraine in those who have only menstrual migraines. As clinical experience suggests these are likely women who do not meet ICHD diagnostic criteria for pure MM, but likely experience the majority of their attacks during the menstrual window and only rare attacks at other times. As both clinic and population diary studies have been limited to three months, it is uncertain whether women who fulfill diagnostic criteria for pure MM in these studies actually occasionally experience attacks outside of their menstrual window. Clinical experience suggests so, but further longitudinal studies are needed to clarify this. Furthermore, if peri-menstrual attacks are truly more severe and burdensome as evidence suggests, there would be a bias in reporting of these events compared to non-menstruation related events. As such, the findings from this study cannot be used to determine if peri-menstrual headaches among women with MM, MAM or MUM are more disabling than headaches that occur at other times in the cycle. However, there is a clear trend of overall greater burden in the MM group than in MAM and both have greater burden than the MUM.

This study has several strengths. First, the study population was population-based and was not subject to the potential selection bias that is a concern with clinicbased samples. That is, our study population was less likely to be prone to selection bias that can occur if women are more likely to seek care because they have migraine headaches associated with menses that are not responsive to treatment. Second, the study population $(\mathrm{N}=1,697)$ was large and representative of the demographics of the U.S. population. Finally, we used well validated instruments to assign migraine diagnosis and asses our primary variables of headache-impact and migraine-related disability.

The primary limitation in this study is that our operational criteria for assigning women to different subgroups differed from the ICHD recommended definitions. Diary data over several months would have been the ideal means of classifying women. Since these data were not available, we relied on self-reported, retrospective data. Our data likely mirror the information that patients will provide to healthcare professionals in clinical practice unless the patient or healthcare professional gather three months of prospective daily diary data. Nonetheless, we assume that self-identification of menstrual migraine status resulted in some misclassification and we acknowledge that we are not approximating the ICHD criteria for menstrual migraine. Rather, this is a descriptive study of women in the US population describing their perception of the relationship between their migraine attacks and their menstrual cycle. Notably, almost a third of MM cases reported seven 
or more headache-days in the past three months. It is likely that a number of these women did not have pure MM according to ICHD, although it is possible if their headaches last three days or if they also have nonmigraine headaches. Furthermore, misclassification of MAM to MM cases is likely to have occurred as outlined above and this would diminish the strength of observed associations (i.e. biased the odds ratio toward the null) because data from MAM would have been falsely mixed in with the MM data. In contrast, misclassification of MM cases as MAM or MUM is likely to have had a negligible effect on assessing differences in disability due to the small size of the group in comparison to other groups.

Furthermore, our definition for MAM ("I am more likely to get headaches with my period but they also occur at other times of the month") was broad and did not require that migraine occur only two days prior or three days after the start of menstruation as is required by ICHD criteria. Individuals could have responded yes to having a "menstrually-associated migraine" even if their migraine occurred on the final three days of their period, which does not match menstrual migraine criteria. It will be useful in the future to determine if peri-menstrual headaches in the MAM group are more disabling than headaches that occur at other times in the cycle. We also did not limit our EM group to those without aura, as is listed in ICHD criteria. Finally, menstrually related migraine has the benefit of being relatively predictable which allows for the preemptive use of therapy or short-term prophylaxis for migraine prevention. While short-term prophylaxis or preemptive use of triptans is not FDA approved, it is a strategy that is used in clinical practice for patients with menstrual related migraine $[8,26,27]$. In the current study we did not report or control for short-term prophylaxis; therefore it is possible that some percentage of the MM group may be well controlled on this therapeutic strategy. Therefore, the disability seen among that group may be reduced due, in some part, to short-term prophylaxis.

\section{Conclusion}

In this large population study representative of the US population, we have shown that most women self-report migraine attacks associated with menstruation and those attacks are associated with impairment including impact on occupational, academic and household responsibilities. When these women are divided into subgroups, those with predominantly peri-menstrual migraines experience the greatest burden. While suggestions for treatment strategies may help, more research will be required to better understand these subgroups given that they account for a significant majority of women with migraine and to determine the best course of treatment for MM and MAM cases and as a result, reduce the burden of migraine associated with menstruation.

\section{Abbreviations}

AMPP: American migraine prevalence and prevention study; MM: Self-reported predominantly menstrual migraine; MAM: Self-reported menstrually associated migraine; MUM: Self-reported menstrually unrelated migraine; ICHD: International classification of headache disorders; MIDAS: Migraine disability assessment scale; HIT: Headache impact test; PMM: Pure menstrual migraine; MRM: Menstrually related migraine; EM: Episodic migraine; LPT: Lost productive time.

\section{Competing interests}

Jelena M. Pavlović, MD, PhD has received honoraria from the American Headache Society and Allergan.

Walter F. Stewart, PhD, received grants from Allergan Pharmaceuticals, Endo Pharmaceuticals, GlaxoSmithKline, Minster, Merck, Inc., Neuralieve, Novartis, OrthoMcNeil, Pfizer, the National Headache Foundation, and the National Institutes of Health.

Christa A. Bruce, MPH, received grants from Allergan Pharmaceuticals and Endo Pharmaceuticals.

Jennifer A. Gorman, MS declared no conflicts of interest for the work. Haiyan Sun, MS declared no conflicts of interest for the work. Dawn C. Buse, PhD has received grant support and honoraria from Allergan/ MAP Pharmaceuticals, Novartis, NuPathe, Zogenix, and the American Headache Society. She is an employee of Montefiore Medical Center, which has received research support funded by Allergan, Colucid, Endo

Pharmaceuticals, GlaxoSmithKline, MAP Pharmaceuticals, Merck, NuPathe, Novartis, Ortho-McNeil, and Zogenix, via grants to the National Headache Foundation.

Richard B. Lipton, MD has received grant support from the National Institutes of Health, the National Headache Foundation, and the Migraine Research Fund. He serves as consultant, advisory board member, or has received honoraria from Alder, Allergan, American Headache Society, Autonomic Technologies, Boston Scientific, Bristol Myers Squibb, Cognimed, Colucid, Eli Lilly, Endo Pharmaceuticals, eNeura Therapeutics, GlaxoSmithKline, MAP, Merck, Novartis, NuPathe, and Pfizer.

\section{Authors' contributions}

JMP drafted the manuscript and revised it for the intellectual content. WFS conceived of the study and participated in its design, coordination, drafting and editing of the manuscript. CAB conceived of the study and participated in its design and coordination and drafting. JAG and HS carried out the statistical analysis and interpretation of the data. DCB conceived of the study and participated in its design and coordination and drafting and revising of the manuscript. RBL conceived of the study, and participated in its design and coordination and helped revise it for the intellectual content. All authors read and approved the final manuscript.

\section{Funding}

The American Migraine Prevalence and Prevention Study is funded through a research grant to the National Headache Foundation from Ortho-McNeil Neurologics, Inc., Titusville, NJ. Additional funding was provided for this manuscript by a grant from ENDO Pharmaceuticals, Chadds Ford, PA to the National Headache Foundation.

\section{References}

1. (2004) The International Classification of Headache Disorders: 2nd edition. Cephalalgia 24 Suppl 1:9-160. http://www.ncbi.nlm.nih.gov/pubmed/14979299.

2. (2013) The International Classification of Headache Disorders, 3rd edition (beta version). Cephalalgia 33:629-808. doi: 10.1177/0333102413485658

3. Couturier EGM, Bomhof MAM, Neven AK, van Duijn NP (2003) Menstrual migraine in a representative Dutch population sample: prevalence, disability and treatment. Cephalalgia 23:302-308

4. Granella F, Sances G, Pucci E, Nappi RE, Ghiotto N, Napp G (2000) Migraine with aura and reproductive life events: a case control study. Cephalalgia 20:701-707

5. MacGregor EA, Brandes J, Eikermann A, Giammarco R (2004) Impact of migraine on patients and their families: the Migraine And Zolmitriptan Evaluation (MAZE) survey-Phase III. Curr Med Res Opin 20:1143-1150, doi:10.1185/030079904125004178

6. MacGregor EA, Frith A, Ellis J, Aspinall L, Hackshaw A (2006) Incidence of migraine relative to menstrual cycle phases of rising and falling estrogen. Neurology 67:2154-2158, doi:10.1212/01.wnl.0000233888.18228.19 
7. Calhoun A, Ford S (2008) Elimination of menstrual-related migraine beneficially impacts chronification and medication overuse. Headache 48:1186-1193

8. Granella F, Sances G, Allais G, Nappi RE, Tirelli A, Benedetto C, Brundu B, Facchinetti F, Nappi G (2004) Characteristics of menstrual and nonmenstrual attacks in women with menstrually related migraine referred to headache centres. Cephalalgia 24:707-716, doi:10.1111/j.1468-2982.2004.00741.x

9. MacGregor EA, Victor TW, Hu X, Xiang Q, Puenpatom RA, Chen W, Campbell JC (2010) Characteristics of menstrual vs nonmenstrual migraine: a post hoc, withinwoman analysis of the usual-care phase of a nonrandomized menstrual migraine clinical trial. Headache 50:528-538, doi:10.1111/j.1526-4610.2010.01625.x

10. Pinkerman B, Holroyd K (2010) Menstrual and nonmenstrual migraines differ in women with menstrually-related migraine. Cephalalgia 30:1187-1194, doi:10.1177/0333102409359315

11. Stewart WF, Lipton RB, Chee E, Sawyer J, Silberstein SD (2000) Menstrual cycle and headache in a population sample of migraineurs. Neurology 55:1517-1523

12. Tepper SJ (2008) Perimenstrual migraines: are they different from migraines in general? Curr Pain Headache Rep 12:463-467

13. Vetvik KG, Russell MB (2011) Are menstrual and nonmenstrual migraine attacks different? Curr Pain Headache Rep 15:339-342, doi:10.1007/s1 1916-011-0212-4

14. Granella F, Sances G, Zanferrari C, Costa A, Martignoni E, Manzoni GC (1993) Migraine without aura and reproductive life events: a clinical epidemiological study in 1300 women. Headache 33:385-389

15. MacGregor EA, Hackshaw A (2004) Prevalence of migraine on each day of the natural menstrual cycle. Neurology 63:351-353

16. Lipton RB, Bigal ME, Diamond M, Freitag F, Reed ML, Stewart WF, AMPP Advisory Group (2007) Migraine prevalence, disease burden, and the need for preventive therapy. Neurology 68:343-349, doi:10.1212/01.wnl.0000252808.97649.21

17. Stewart WF, Lipton RB, Celentano DD, Reed ML (1992) Prevalence of migraine headache in the United States. Relation to age, income, race, and other sociodemographic factors. JAMA 267:64-69

18. Lipton RB, Stewart WF, Diamond S, Diamond ML, Reed M (2001) Prevalence and burden of migraine in the United States: data from the American Migraine Study II. Headache 41:646-657

19. Lipton RB, Diamond S, Reed M, Diamond ML, Stewart WF (2001) Migraine Diagnosis and Treatment: Results From the American Migraine Study II. Headache J Head Face Pain 41:638-645, doi:10.1046/j.1526 4610.2001.041007638.x

20. Stewart WF, Lipton RB, Kolodner KB, Sawyer J, Lee C, Liberman JN (2000) Validity of the Migraine Disability Assessment (MIDAS) score in comparison to a diarybased measure in a population sample of migraine sufferers. Pain 88:41-52

21. Kosinski M, Bayliss MS, Bjorner JB, Ware JE Jr, Garber WH, Batenhorst A, Cady R, Dahlöf CG, Dowson A, Tepper S (2003) A six-item short-form survey for measuring headache impact: the HIT-6. Qual Life Res 12:963-974

22. Stewart WF, Ricci JA, Chee E, Morganstein D, Lipton R (2003) Lost productive time and cost due to common pain conditions in the US workforce. JAMA 290:2443-2454, doi:10.1001/jama.290.18.2443

23. Stewart WF, Wood GC, Razzaghi H, Reed ML, Lipton RB (2008) Work impac of migraine headaches. J Occup Environ Med 50:736-745, doi:10.1097/ JOM.0b013e31818180cb

24. Dowson AJ, Kilminster SG, Salt R, Clark M, Bundy MJ (2005) Disability associated with headaches occurring inside and outside the menstrual period in those with migraine: a general practice study. Headache 45:274-282, doi:10.1111/j.1526-4610.2005.05064.x

25. Diamond ML, Cady RK, Mao L, Biondi DM, Finlayson G, Greenberg SJ, Wrigh P (2008) Characteristics of migraine attacks and responses to almotriptan treatment: a comparison of menstrually related and nonmenstrually related migraines. Headache 48:248-258, doi:10.1111/j.1526-4610.2007.01019.x

26. Macgregor EA (2009) Menstrual migraine: therapeutic approaches. Ther Adv Neurol Disord 2:327-336, doi:10.1177/1756285609335537

27. Silberstein SD, Elkind AH, Schreiber C, Keywood C (2004) A randomized trial of frovatriptan for the intermittent prevention of menstrual migraine. Neurology 63:261-269

\section{Submit your manuscript to a SpringerOpen ${ }^{\odot}$ journal and benefit from:}

- Convenient online submission

- Rigorous peer review

- Immediate publication on acceptance

- Open access: articles freely available online

- High visibility within the field

- Retaining the copyright to your article

Submit your next manuscript at $>$ springeropen.com 